\title{
Antibiotic resistance rates among bacterial isolates from infected patients of intensive care unit over the period (2011-2016) in Bursa, Turkey
}

\section{Abstract}

Background: The aims of this study were to evaluate the microbiological data for effective infection control and specific antimicrobial stewardship, and to report the epidemiology of intensive care unit (ICU)-acquired infections and the local antimicrobial resistance of pathogens

Methods: Data were collected between January 2011 and October 2016 from the cardiology and cardiovascular surgery patients. The identification and antimicrobial susceptibility analyses of clinical pathogenic isolates were determined by the automatic device system.

Results: Klebsiella pneumoniae was detected increasingly and significantly ( $p=0.048$ ) resistant to imipenem (IMP). Acinetobacter baumanii was found increasingly and significantly $(p=0.045, p=0.030, p=0.006$, $p=0.027$ ) resistant to amikacin $(A N)$, gentamicin $(G N)$, trimethoprimsulfamethoxazole (SXT) and tetracycline (TE), respectively. There was a significantly decrease in the resistant rates of IMP, piperacillin-tazobactam (TPZ), meropenem (MEM) and ciprofloxacin (CIP) for Pseudomonas spp. $(p=0.048)$. All gram-negative bacteria isolates showed significantly increasing resistance rates to cefepime (CEP) $(p=0.015)$. Also, all gram-positive bacteria demonstrated significantly decreasing resistance rates to teicoplanin (TEC) $(p=0.034)$.

Conclusion: This study suggets that inadequacy of infection control measures such as hand hygiene and patient isolation may have contributed to increasing the rates of carbapenem-resistant $K$. pnemoniae $(\mathrm{CR}-\mathrm{KP})$ isolates in recent years.
Sanem Karadag Gecgel ${ }^{1}$, Necmiye Demircan ${ }^{2}$

1 Departments of Microbiology and Clinical Microbiology, University of Health Sciences, Yuksek Ihtisas Training and Research Hospital, Bursa, Turkey.

2 Departments of Infectious Diseases and Clinical Microbiology, University of Health Sciences, Yuksek Ihtisas Training and Research Hospital, Bursa, Turkey.

\section{Contact information:}

Sanem Karadag Gecgel.

Đ sanemkaradag@yahoo.com 


\section{Introduction}

The antimicrobial stewardship in intensive care unit (ICU) is highly important measurement to control antimicrobial resistance in most hospital. There are two main problems to this issue. Inappropriate usage of antibiotic therapy and poor concordance to the infection control policy. Both can lead to the occurrence of resistant pathogens by various molecular mechanisms such as mutation, gene transfer and selection of the resistant strains [1, 2]. Recently, most ICU-acquired infection are due to pathogens like methicillin-resistant Staphylococci, multidrug-resistant Pseudomonas aeruginosa and Acinetobacter species. Additionaly, carbapenem resistant $K$. pneumoniae (CR-KP) are detected more frequently in the recent years worldwide [3-5]. Therefore, awareness about the current local epidemiology and antimicrobial susceptibility of bacterial isolates is an important factor in prescribing true empiric antibiothreapy in ICU $[6,7]$. Despite this fact, medical treatment options of multidrug-resistant pathogens are limited [8].

The aim of this study is to report the epidemiology of ICU-acquired infections and the local antimicrobial resistance of microorganism isolates over a 6- year period.

\section{Materials and Methods}

\section{Patients}

Data were collected between January 2011-October 2016, retrospectively, from admitted adult cardiology and cardiovascular surgery (CVS) patients at Bursa Yuksek Ihtisas Training and Research Hospital, Turkey. This hospital is a tertiary care hospital serving a 3-million population region. ICU-acquired infections were diagnosed after 48 hours of ICU admission according to Centers for Disease Control and Prevention guidelines [9]. Piperacillin-tazobactam, imipenem, meropenem antibiotics were frequently used in treatment of ICU-acquired infection caused by gram-negative bacteria and vancomycin, linezolid, daptomycin antibiotics were frequently used in treatment of ICU-acquired infection caused by gram-positive bacteria.

\section{Microbiological analyses}

Blood, urine, wound, sputum, tracheal aspirate and other clinical samples were collected from the patients who were prediagnosed with ICU-acquired infections. Cultivation of specimens were made and microbiological tests were used for identification of isolates. Bacterial isolates from blood cultures were detected using BACTEC 9240 (Becton Dickinson, USA). Identification and antimicrobial susceptibility analyses of pathogens were determined by the automatic device system (MicroScan Walkaway, Beckman Coulter, USA). The following antibiotics were used for gram-negative microorganisms; amikacin (AN), amoxicillin-clavulanate (AMC), gentamicin $(\mathrm{GN})$, imipenem (IMP), piperacillin-tazobactam (TPZ), cefepime (CEP), cefotaxime (CTX), trimethoprim-sulfamethoxazole (SXT), meropenem (MEM), ciprofloxacin (CIP), ceftazidime (CAZ), levofloxacin (LEV), tetracycline (TE), cefazolin (CZ) antimicrobial resistance ratios were established. For gram-positive microorganisms; ampicillin (AM), ciprofloxacin (CIP), teicoplanin (TEC), tetracycline (TE), trimethoprimsulfamethoxazole (SXT), vancomycin (VA), gentamicin (GN). All antimicrobial resistance ratios were determined according to the minimal inhibitor concentration (MIC) values of the current EUCAST criteria. Methicillin resistant Staphylococcus species was assessed according to cefoxitin MIC values. Candida species was differentiated by APIc AUX kit (bioMe'rieux, France).

\section{Statistical analyses}

Data were analyzed by SPSS program version 21.0 with Fisher's exact chi-square test and Pearson chisquare test. Differences were accepted statistically significant at $p<0.05$. 


\section{Results}

The distribution of recovered pathogens in ICUs over a 6-year period is demonstrated in Table 1. Acinetobacter baumanii and coagulase-negative Staphylococcus (CNS) were the most common pathogens recovered. The distribution of resistance rates of recoverd pathogens through three-year periods was determined. Antibiotic resistance rates of microorganisms between 2011-2012-2013 and 2014-
2015-2016 were combined and the total resistance rates in both periods were compared. A comparison between resistance rates in gram-negative pathogens during a 6 -year period is demonstrated in Table $2, \mathbf{3}$. A second comparison between resistance rates in gram-positive bacteria over a 6 -year period is shown in Table 4.

Table 1. Distribution of ICU-acquired pathogens over the 6-year period (2011-2016).

\begin{tabular}{|c|c|c|c|c|c|c|c|c|c|c|c|c|}
\hline \multirow{2}{*}{ Microorganism isolates } & \multicolumn{2}{|c|}{2011} & \multicolumn{2}{|c|}{2012} & \multicolumn{2}{|c|}{2013} & \multicolumn{2}{|c|}{2014} & \multicolumn{2}{|c|}{2015} & \multicolumn{2}{|c|}{2016} \\
\hline & No. & $\%$ & No. & $\%$ & No. & $\%$ & No. & $\%$ & No. & $\%$ & No. & $\%$ \\
\hline Acinetobacter baumannii & 23 & 23.7 & 8 & 10 & 18 & 18.75 & 18 & 20.93 & 0 & & 9 & 16.36 \\
\hline Acinetobacter spp. & 2 & 2.1 & 10 & 12.5 & 7 & 7.29 & 10 & 11.62 & 16 & 21.33 & 6 & 10.90 \\
\hline Candida albicans & 0 & & 2 & 2.5 & 3 & 3.12 & 4 & 4.65 & 0 & & 0 & \\
\hline Candida spp. & 6 & 6.2 & 2 & 2.5 & 5 & 5.20 & 3 & 3.48 & 1 & 1.33 & 0 & \\
\hline Citrobacter spp. & 1 & 1 & 0 & & 2 & 2.08 & 0 & & 1 & 1.33 & 2 & 3.63 \\
\hline Enterococcus spp. & 0 & & 4 & 5 & 3 & 3.12 & 2 & 2.32 & 0 & & 2 & 3.63 \\
\hline Enterobacter spp. & 1 & 1 & 2 & 2.5 & 0 & & 0 & & 1 & 1.33 & 0 & \\
\hline Enterobacter aerogenes & 1 & 1 & 1 & 1.25 & 1 & 1.04 & 5 & 5.81 & 0 & & 0 & \\
\hline Enterobacter cloacae & 0 & & 4 & 5 & 1 & 1.04 & 2 & 2.32 & 0 & & 1 & 1.81 \\
\hline Enterococcus faecalis & 5 & 5.2 & 2 & 2.5 & 8 & 8.33 & 4 & 4.65 & 3 & 4 & 4 & 7.27 \\
\hline Enterococcus faecium & 3 & 3.1 & 0 & & 3 & 3.12 & 1 & 1.16 & 1 & 1.33 & 1 & 1.81 \\
\hline Escherichia coli & 11 & 11.3 & 5 & 6.25 & 6 & 6.25 & 3 & 3.48 & 10 & 13.33 & 5 & 9.09 \\
\hline Coagulase-negative staphylococci & 8 & 8. 3 & 17 & 21.25 & 18 & 18.75 & 9 & 10.46 & 12 & 16 & 9 & 16.36 \\
\hline Klebsiella oxytoca & 0 & & 1 & 1.25 & 0 & & 0 & & 0 & & 0 & \\
\hline Klebsiella pneumoniae & 8 & 8.2 & 3 & 3.75 & 2 & 2.08 & 8 & 9.30 & 3 & 4 & 5 & 9.09 \\
\hline Klebsiella spp. & 0 & & 3 & 3.75 & 0 & & 2 & 2.32 & 6 & 8 & 0 & \\
\hline Morganella morganii & 0 & & 0 & & 0 & & 0 & & 1 & 1.33 & 1 & 1.81 \\
\hline Pseudomonas aeruginosa & 2 & 2.1 & 3 & 3.75 & 10 & 10.41 & 4 & 4.65 & 3 & 4 & 3 & 5.45 \\
\hline Pseudomonas spp. & 0 & & 0 & & 2 & 2.08 & 3 & 3.48 & 2 & 2.66 & 0 & \\
\hline Proteus mirabilis & 0 & & 0 & & 1 & 1.04 & 1 & 1.16 & 0 & & 0 & \\
\hline Providencia spp. & 1 & 1. & 0 & & 0 & & 0 & & 0 & & 0 & \\
\hline Serratia marcescens & 6 & 6.2 & 0 & & 2 & 2.08 & 0 & & 1 & 1.33 & 1 & 1.81 \\
\hline Staphylococcus aureus & 16 & 16.5 & 13 & 16.25 & 1 & 1.04 & 7 & 8.13 & 13 & 17.33 & 6 & 10.90 \\
\hline Streptococcus pneumoniae & 1 & 1 & 0 & & 1 & 1.04 & 0 & & 1 & 1.33 & 0 & \\
\hline Stenotrophomonas maltophilia & 1 & 1 & 0 & & 2 & 2.08 & 0 & & 0 & & 0 & \\
\hline Streptococcus spp. & 1 & 1 & 0 & & 0 & & 0 & & 0 & & 0 & \\
\hline Total & 97 & 100 & 80 & 100 & 96 & 100 & 86 & 100 & 75 & 100 & 55 & 100 \\
\hline
\end{tabular}


Table 2. Comparison of the antimicrobial resistance rates in different gram negative pathogens over two periods (2011-2013 versus 2014-2016).

\begin{tabular}{|c|c|c|c|c|c|c|c|c|c|c|c|c|c|c|c|}
\hline \multirow{2}{*}{ Microorganism } & \multirow{2}{*}{ Period } & \multicolumn{2}{|c|}{ Amikacin } & \multicolumn{2}{|c|}{ Amoxicillin/Clavulanate } & \multicolumn{2}{|c|}{ Gentamicin } & \multicolumn{2}{|c|}{ Imipenem } & \multicolumn{2}{|c|}{ Piperacillin/Tazobactam } & \multicolumn{2}{|c|}{ Cefepime } & \multicolumn{2}{|c|}{ Cefotaxime } \\
\hline & & $\%$ & $p^{*}$ & $\%$ & $p$ & $\%$ & $p$ & $\%$ & $p$ & $\%$ & $p$ & $\%$ & $p$ & $\%$ & $p$ \\
\hline \multirow[t]{2}{*}{ Enterobacter cloacae } & $2011-2013$ & 0.0 & & 100.0 & & 0.0 & & 0.0 & & 20.0 & \multirow{2}{*}{1.00} & 0.0 & \multirow{2}{*}{0.143} & 25.0 & \multirow{2}{*}{1.00} \\
\hline & $2014-2016$ & 0.0 & & 100.0 & & 0.0 & & 0.0 & & 0.0 & & 66.7 & & 33.3 & \\
\hline \multirow[t]{2}{*}{ E. coli } & $2011-2013$ & 9.1 & \multirow{2}{*}{0.642} & 93.3 & \multirow{2}{*}{0.314} & 47.6 & \multirow{2}{*}{0.180} & 0.0 & \multirow{2}{*}{0.450} & & & & & 37.5 & \multirow{2}{*}{1.00} \\
\hline & $2014-2016$ & 16.7 & & 66.7 & & 22.2 & & 5.6 & & & & & & 40.0 & \\
\hline \multirow[t]{2}{*}{ Klebsiella pneumoniae } & $2011-2013$ & 7.7 & \multirow{2}{*}{0.343} & 50.0 & \multirow{2}{*}{1.00} & 69.2 & \multirow{2}{*}{1.00} & 0.0 & \multirow{2}{*}{0.048} & 50.0 & \multirow{2}{*}{1.00} & & & 62.5 & \multirow{2}{*}{1.00} \\
\hline & $2014-2016$ & 25.0 & & 50.0 & & 62.5 & & 31.2 & & 50.0 & & & & 50.0 & \\
\hline \multirow[t]{2}{*}{ Klebsiella spp. } & $2011-2013$ & & & & & 66.7 & & 0.0 & & & & & & 100.0 & \\
\hline & $2014-2016$ & & & & & 37.5 & 0.545 & 0.0 & & & & & & 100.0 & \\
\hline Serratia marcescens & $2011-2013$ & 0.0 & & 100.0 & & 0.0 & & 0.0 & & & & 0.0 & & 12.5 & \\
\hline & $2014-2016$ & 0.0 & & 100.0 & & 0.0 & & 0.0 & & & & 0.0 & & 0.0 & 1.00 \\
\hline Pseudomonas aeruginosa & $2011-2013$ & 7.1 & & & & 7.1 & & 33.3 & & 0.0 & & & & & \\
\hline & $2014-2016$ & 14.3 & 1.00 & & & 28.6 & 0.521 & 0.0 & 0.262 & 0.0 & & & & & \\
\hline Acinetobacter baumanii & $2011-2013$ & 83.0 & & & & 67.6 & 0.030 & 97.8 & & & & & & 100.0 & \\
\hline & 2014-2016 & 100.0 & 0.045 & & & 92.6 & & 96.3 & 1.00 & & & & & 100.0 & \\
\hline Acinetobacter spp. & $2011-2013$ & 33.3 & & & & 78.9 & & 76.5 & & & & 94.7 & & 94.7 & \\
\hline & 2014-2016 & 59.1 & 0.184 & & & 59.4 & 0.222 & 81.2 & 0.121 & & & 100.0 & 1.00 & 100.0 & 1.00 \\
\hline Enterobacter aerogenes & $2011-2013$ & 0.0 & & 100.0 & & 0.0 & & 0.0 & & 0.0 & & 0.0 & & 0.0 & \\
\hline & $2014-2016$ & 0.0 & & 100.0 & & 40.0 & 0.464 & 0.0 & & 0.0 & & 60.0 & 0.196 & 0.0 & \\
\hline Citrobacter spp. & $2011-2013$ & 33.3 & & & & 33.3 & & 33.3 & & & & & & & \\
\hline & $2014-2016$ & 0.0 & 1.00 & & & 33.3 & 1.00 & 0.0 & 1.00 & & & & & & \\
\hline Pseudomonas spp. & $2011-2013$ & & & & & & & 100.0 & & 100.0 & & & & & \\
\hline & $2014-2016$ & & & & & & & 0.0 & 0.048 & 0.0 & 0.048 & & & & \\
\hline Proteus mirabilis & $2011-2013$ & 0.0 & & 0.0 & & & & & & & & 0.0 & 1.00 & & \\
\hline & 2014-2016 & 100.0 & 1.00 & 0.0 & & & & & & & & 100.0 & & & \\
\hline Gram Negative & $2011-2013$ & 37.5 & & 92.9 & & 50.0 & & 47.1 & & 36.0 & & 53.8 & & 75.4 & \\
\hline Microorganisms & $2014-2016$ & 46.2 & 0.190 & 73.9 & 0.057 & 53.2 & 0.620 & 46.0 & 0.902 & 25.7 & 0.569 & 84.6 & 0.015 & 76.5 & 1.00 \\
\hline
\end{tabular}


Table 3. Comparison of antimicrobial resistance rates in different gram negative pathogens over two periods (2011-2013 versus 2014-2016)

\begin{tabular}{|c|c|c|c|c|c|c|c|c|c|c|c|c|c|c|c|}
\hline \multirow{2}{*}{ Microorganism } & \multirow{2}{*}{ Period } & \multicolumn{2}{|c|}{ Trimethoprim/Sulfamethoxazole } & \multicolumn{2}{|c|}{ Meropenem } & \multicolumn{2}{|c|}{ Ciprofloxacin } & \multicolumn{2}{|c|}{ Ceftazidim } & \multicolumn{2}{|c|}{ Levofloxacin } & \multicolumn{2}{|c|}{ Tetracycline } & \multicolumn{2}{|c|}{ Cefazolin } \\
\hline & & $\%$ & $p *$ & $\%$ & $p$ & $\%$ & $p$ & $\%$ & $p$ & $\%$ & $p$ & $\%$ & $p$ & $\%$ & $p$ \\
\hline \multirow[t]{2}{*}{ Enterobacter cloacae } & $2011-2013$ & 20.0 & \multirow{2}{*}{1.00} & \multirow{4}{*}{$\begin{array}{l}0.0 \\
5.6\end{array}$} & \multirow{4}{*}{0.450} & & & & & & & & & & \\
\hline & 2014-2016 & 0.0 & & & & & & & & & & & & & \\
\hline \multirow[t]{2}{*}{ E. coli } & $2011-2013$ & 76.5 & \multirow{2}{*}{1.00} & & & & & & & & & & & & \\
\hline & $2014-2016$ & 80.0 & & & & & & & & & & & & & \\
\hline \multirow[t]{2}{*}{ Klebsiella pneumoniae } & $2011-2013$ & 61.5 & \multirow{2}{*}{0.714} & 0.0 & \multirow{2}{*}{0.096} & 61.5 & \multirow{2}{*}{0.673} & & & & & & & & \\
\hline & 2014-2016 & 68.8 & & 30.8 & & 50.0 & & & & & & & & & \\
\hline \multirow[t]{2}{*}{ Klebsiella spp. } & $2011-2013$ & 33.3 & \multirow{2}{*}{0.491} & & & 66.7 & \multirow{2}{*}{1.00} & & & & & & & & \\
\hline & $2014-2016$ & 75.0 & & & & 62.5 & & & & & & & & & \\
\hline \multirow[t]{2}{*}{ Serratia marcescens } & $2011-2013$ & & & 0.0 & & & & 0.0 & & & & & & & \\
\hline & $2014-2016$ & & & 0.0 & & & & 50.0 & 0.22 & & & & & & \\
\hline \multirow[t]{2}{*}{ Pseudomonas aeruginosa } & $2011-2013$ & & & 33.3 & \multirow{2}{*}{0.345} & 20.0 & \multirow{2}{*}{1.00} & 46.7 & & 0.0 & \multirow{2}{*}{1.00} & & & & \\
\hline & $2014-2016$ & & & 10.0 & & 25.0 & & 60.0 & 0.68 & 16.7 & & & & & \\
\hline Acinetobacter baumanii & $2011-2013$ & 75.5 & & 98.0 & & 95.9 & & 95.7 & & 100.0 & & 76.6 & & & \\
\hline & $2014-2016$ & 100.0 & 0.06 & 100.0 & 1.00 & 100.0 & 0.536 & 100.0 & 0.530 & 100.0 & & 100.0 & 0.021 & & \\
\hline Acinetobacter spp. & $2011-2013$ & & & 83.3 & & 100.0 & & 100.0 & & 100.0 & & & & & \\
\hline & $2014-2016$ & & & 96.2 & 0.289 & 100.0 & & 100.0 & & 100.0 & & & & & \\
\hline Enterobacter aerogenes & $2011-2013$ & 0.0 & 0196 & 0.0 & & & & 0.0 & & & & & & 100.0 & \\
\hline & $2014-2016$ & 60.0 & 0.190 & 0.0 & & & & 0.0 & & & & & & 100.0 & \\
\hline Citrobacter spp. & $2011-2013$ & 33.3 & 100 & & & 0.0 & & & & & & & & & \\
\hline & $2014-2016$ & 100.0 & 1.00 & & & 0.0 & & & & & & & & & \\
\hline Pseudomonas spp. & $2011-2013$ & & & 100.0 & & 100.0 & & & & & & & & & \\
\hline & $2014-2016$ & & & 0.0 & 0.048 & 0.0 & 0.048 & & & & & & & & \\
\hline Proteus mirabilis & $2011-2013$ & & & & & & & & & & & & & 0.0 & \\
\hline & 2014-2016 & & & & & & & & & & & & & 0.0 & \\
\hline Gram Negative & $2011-2013$ & 64.3 & & 52.2 & & 75.5 & & 76.3 & & 88.1 & & 78.0 & & 100.0 & \\
\hline Microorganisms & $2014-2016$ & 76.5 & 0.123 & 50.0 & 0.191 & 81.2 & 0.386 & 86.7 & 0.11 & 85.3 & $0.15 /$ & 100.0 & 0.055 & 87.5 & 0.381 \\
\hline
\end{tabular}


Table 4. Comparison of antimicrobial susceptibility rates in different gram positive pathogens over two periods (2011-2013 versus 2014-2016).

\begin{tabular}{|c|c|c|c|c|c|c|c|c|c|c|c|c|c|c|c|}
\hline \multirow{2}{*}{ Microorganism } & \multirow{2}{*}{ Period } & \multicolumn{2}{|c|}{ Ampicillin } & \multicolumn{2}{|c|}{ Ciprofloxacin } & \multicolumn{2}{|c|}{ Teicoplanin } & \multicolumn{2}{|c|}{ Tetracycline } & \multicolumn{2}{|c|}{ Trimethoprim/Sulfamethoxazole } & \multicolumn{2}{|c|}{ Vancomycin } & \multicolumn{2}{|c|}{ Gentamicin } \\
\hline & & $\%$ & $P^{*}$ & $\%$ & $p$ & $\%$ & $p$ & $\%$ & $p$ & $\%$ & $\mathrm{p}$ & $\%$ & $p$ & $\%$ & $p$ \\
\hline \multirow[t]{2}{*}{ Staphylococcus aureus } & $2011-2013$ & 100.0 & \multirow{2}{*}{0.248} & 75.0 & \multirow{2}{*}{0.111} & 0.0 & & 53.3 & \multirow{2}{*}{0.693} & 33.3 & \multirow{2}{*}{0.290} & 0.0 & & & \\
\hline & 2014-2016 & 81.2 & & 38.5 & & 0.0 & & 42.9 & & 15.4 & & 0.0 & & & \\
\hline \multirow[t]{2}{*}{ Enterococcus faecalis } & $2011-2013$ & 0.0 & \multirow{2}{*}{0.169} & 25.0 & \multirow{2}{*}{0.315} & 10.0 & \multirow{2}{*}{0.476} & 70.0 & \multirow{2}{*}{0.659} & & & 0.0 & & & \\
\hline & $2014-2016$ & 18.2 & & 62.5 & & 0.0 & & 54.5 & & & & 0.0 & & & \\
\hline Enterococcus spp. & $2011-2013$ & 50.0 & 1.00 & & & & & 100.0 & & & & 0.0 & & & \\
\hline \multirow[t]{2}{*}{$\mathrm{CNS}^{* *}$} & $2011-2013$ & 100.0 & \multirow{2}{*}{0.492} & & & 7.1 & \multirow{2}{*}{0.261} & 23.3 & \multirow{2}{*}{0.547} & 48.8 & \multirow{2}{*}{1.00} & 0.0 & & 46.3 & \multirow{2}{*}{0.474} \\
\hline & $2014-2016$ & 93.1 & & & & 0.0 & & 33.3 & & 52.4 & & 0.0 & & 56.7 & \\
\hline \multirow[t]{2}{*}{ Enterococcus faecium } & $2011-2013$ & 100.0 & & & & 33.0 & \multirow{2}{*}{0.500} & & & & & 33.0 & \multirow{2}{*}{0.500} & & \\
\hline & $2014-2016$ & 100.0 & & & & 0.0 & & & & & & 0.0 & & & \\
\hline \multirow{2}{*}{$\begin{array}{l}\text { Gram positive } \\
\text { microorganisms }\end{array}$} & $2011-2013$ & 32.0 & \multirow{2}{*}{0.747} & 55.0 & \multirow{2}{*}{0.758} & 6.8 & \multirow{2}{*}{0.034} & 42.5 & 1.00 & 42.5 & 0.833 & 1.9 & 0.524 & 46.3 & 0.474 \\
\hline & 2014-2016 & 37.5 & & 47.6 & & 0.0 & & 43.9 & & 38.2 & כתס. & 0.0 & 0.324 & 56.7 & 0.414 \\
\hline
\end{tabular}

\section{Discussion}

Hospitalization in ICU is an important risk factor to acquire infection with carbapenem resistant $K$. pneumoniae (CR-KP), because of usage of many invasive devices such as urinary catheters, central venous catheters, mechanical ventilation and the possibility of transmission infection between patients. Additionaly, broad-spectrum antimicrobial drugs such as carbapenems are frequently used in ICUs and these drugs are known to contribute for emergence of CR-KP [10-12]. In our study,
K. pneumoniae was detected increasingly and significantly resistant to imipenem, because our tertiary care hospital admitted cardiology and cardiovascular surgery patients (CVS) from referral hospitals to our ICUs. We suspect that these patients have contributed for spread of CR-KP in our ICUs. In addition, the insufficient isolation of infected patients and inadequate ventilation in our ICUs have increased rates of infection and isolation of resistant pathogens among patients during the two compared periods (2014-2016 versus
2011-2013) as shown in Table 2-4. Also, similar increasing rates were observed in relation to use of urinary catheterization and the length period of usage it. Both these two factors were most probably increased infection with CR-KP in our intensive care units.

In our study, multidrug-resistant $A$. baumanii isolates were the most common cause of infections among our patients in ICUs as it has been recoded worldwide [13-16]. The increase in antibiotic resistance rates among $A$. baumanii isolates 
was significant (Table 2-3). It has been reported that carbapenem-resistant Acinetobacter isolates can be increased rapidly and become a significant problem in intensive care unit patients. Additionaly, rates of resistant Acinetobacter strains are observed to be higher in countries where carbapenems are intensely used $[17,18]$.

Studies have also shown an increasing carbapenem-resistance in $P$. aeruginosa isolates from ICU-acquired infections [19-21]. In our study, there was a significantly decrease in the resistant rates of Pseudomonas spp. and P. aeruginosa to IMP, TPZ, MEM and CIP, but both were not istatistically significant. In addition, decreased rates of resistance to IMP and MEM were detected in all gram-negative pathogens and these were not statistically significant (Table 2-3). The reason for this decrease may be due the fact that in our hospital, our physicians used less empirical treatment in ICU-acquired infections and they are preferring to apply mostly treatment based on culture results. This approach may has led to reduce the occcurrence of carbapenem-resistant pathogens in our hospital. It is also impotant to note that our hospital's microbiology laboratory provided 24-hour service, and offer a rapid assessment of positive blood cultures and the result of gram-staining.

In our hospital, physcians are prefering treatment of gram-positive bacterial infections with vancomycin, daptomycin and linezolid instead of teicoplanin. Despite this treatment approch, there was overall no change in rates of resistant among all gram-positive bacteria to these 3 drugs, but there was statistically significant decreased in resistant to TEC (Table 4). However, various studies have shown that teicoplanin could theoretically represent an acceptable alternative drug, since it has comparable efficacy compared to vancomycin in various disease conditions and excellant bone diffusion [22]. Extensive use of teicoplanin in ICU patienst may result in development of increased resistance rates in gramposive bacteria, whereas the usage of vancomycin occasionally during empirical therapy, and according to MIC values were the main reasons for not increasing vancomycin resistance rates in gram-positive bacteria in this study [23-26].

In conclusion, this study showes that close cooperation between microbiology laboratory and physcians has been associated with decrease in carbapenem and vancomycin resistance rates in pathogens recovered from ICU's patients.

\section{Acknowledgements}

We thank the dedicated staff members at the Clinical Microbiology, Bursa Yuksek Ihtisas Training and Research Hospital, for their technical support.

\section{Funding}

There is no relevant sources of funding for this study

\section{Conflicts of interest}

There are no conflicts of interest for this study.

\section{Ethical approval}

Not required.

\section{References}

1. Timsit JF, Harbarth S, Carlet J. De-escalation as a potential way of reducing antibiotic use and antimicrobial resistance in ICU. Intensive Care Med 2014; 40: 1580-82.

2. Kaki R, Elligsen M, Walker S, Simor A, Palmay L, Daneman N. Impact of antimicrobial stewardship in critical care: a systematic review. Antimicrob Chemother 2011; 66: 1223-30.

3. Mitharwal SM, Yaddanapudi S, Bhardwaj N, Gautam V, Biswal M, Yaddanapudi L. Intensive care unit-acquired infections in a tertiary care hospital: An epidemiologic survey and influence on patient outcomes. Am J Infect Control 2016; 44: e113-7.

4. Kousouli E, Zarkotou O, Politi L, Polimeri K, Vrioni G, ThemeliDigalaki K, Tsakris A, Pournaras S. Infection control interventions affected by resource shortages: impact on the incidence of bacteremias caused by carbapenem-resistant pathogens. Eur J Clin Microbiol Infect Dis 2017 Sep 6. doi: 10.1007/s10096-0173098-1.

5. Papadimitriou-Olivgeris M, Fligou F, Spiliopoulou A, Koutsileou K, Kolonitsiou F, Spyropoulou A, Zotou A, Marangos M, Anastassiou ED, Christofidou M, Spiliopoulou I. Risk factors and predictors of carbapenem-resistant Pseudomonas aeruginosa and Acinetobacter baumannii mortality in critically ill bacteraemic patients over a 6-year period (2010-15): antibiotics do matter. J Med Microbiol 2017; 66: 1092-1101. 
6. Hughes JS, Hurford A, Finley RL, Patrick DM, Wu J, Morris AM. How to measure the impacts of antibiotic resistance and antibiotic development on empiric therapy: new composite indices. BMJ Open 2016; 6: e012040.

7. Sydnor ERM, Perl TM. Hospital epidemiology and infection control in acute care settings. Clin Microbiol Rev 2011; 24: 14173 .

8. WHO: World Health Organization. Antimicrobial resistance: global report on surveillance. 2014. http://www.who.int/ drugresistance/documents/surveillancereport/en.

9. Akcay SS, Inan A, Cevan S, Ozaydin AN, Cobanoglu N, Ozyurek SC, Aksaray S. Gram-negative bacilli causing infections in an intensive care unit of a tertiary care hospital in Istanbul, Turkey. Infect Dev Ctries 2014; 8: 597-604.

10. Yang D, Xıe Z, Xın X, Xue W, Zhang M. A model for predicting nosocomial carbapenem resistant Klebsiella pneumoniae infection. Biomed Rep 2016; 5: 501-5.

11. Brusselaers $N$, Vogelaers $D$, Blot $S$. The rising problem of antimicrobial resistance in the intensive care unit. Ann Intensive Care 2011; 1: 47.

12. Hu Y, Ping $Y$, Li L, Xu H, Yan $X$, Dai H. A retrospective study of risk factors for carbapenem-resistant Klebsiella pneumoniae acquisition among ICU patients. J Infect Dev Ctries 2016; 10 : 208-13.

13. Ho YH, Tseng CC, Wang LS, Chen YT, Ho GJ, Lin TY, Wang LY, Chen LK. Application of bacteriophage-containing aerosol against nosocomial transmission of carbapenem-resistant Acinetobacter baumannii in an intensive care unit. Plos one 2016; 11: e0168380.

14. Izadpour F, Ranjbari N, Aramesh MR, Moosavian M, ShahAli S, Larki F, Tabesh H, Morvaridi A. An investigation of antibacterial resistance patterns among Acinetobacter baumannii and Pseudomonas aeruginosa isolates collected from intensive care units of a university-affiliated hospital in Ahvaz, Iran. Jundishapur J Microbiol 2016; 9: e35624.

15. Agodi A, Barchitta M, Quattrocchi A, Maugeri A, Aldisio E, Marchese AE, Mattaliano AR, Tsakris A. Antibiotic trends of Klebsiella pneumoniae and Acinetobacter baumannii resistance indicators in an intensive care unit of Southern Italy, 2008-2013. Antimicrob Resist Infect Control 2015; 4: 43.

16. Meric M, Baykara N, Aksoy S, Kol IO, Yilmaz G, Beyazit N, Mete $B$, Vahaboglu $\mathrm{H}$; Epidemiology and risk factors of intensive care unit-acquired infections: a prospective multicentre cohort study in a middle-income country. Singapore Med J 2012; 53: 260-3.

17. MacVane SH. Antimicrobial resistance in the intensive care unit: A focus on gram-negative bacterial infections. J Intensive care Med 2017; 32: 25-37.

18. Aziz Ogutlu, Ertugrul Guclu, Oguz Karabay, Aylin Calica Utku, Nazan Tuna, Mehmet Yahyaoglu. Effects of Carbapenem consumption on the prevalence of Acinetobacter infection in intensive care unit patients. Ann Clin Microbiol Antimicrob 2014; 13 : 7.

19. Paramythiotou E, Routsi C. Association between infections caused by multidrug-resistant gram-negative bacteria and mortality in critically ill patients. World J Crit Care Med 2016; 5: $111-20$.
20. Aykan ŞB, Çiftci iH. Changes in antibiotic resistance of Pseudomonas aeruginosa isolates over the past 11 years in Turkey: a meta-analysis. Mikrobiyol Bul 2015; 49: 352-65.

21. Zhang $X, G u$ B, Mei $Y$, Wen $Y$, Xia W. Increasing resistance rate to carbapenem among blood culture isolates of Klebsiella pneumoniae, Acinetobacter baumannii and Pseudomonas aeruginosa in a university-affiliated hospital in China, 20042011. J Antibiot (Tokyo) 2015; 68: 115-20.

22. Peeters O, Ferry T, Ader F, Boibieux A, Braun E, Bouaziz A, Karsenty J, Forestier E, Laurent F, Lustig S, Chidiac C, Valour F and Lyon BJI study group. Teicoplanin-based antimicrobial therapy in Staphylococcus aureus bone and joint infection: tolerance, efficacy and experience with subcutaneous administration. BMC Infect Dis 2016; 16: 622.

23. Jones RN. Key considerations in the treatment of complicated staphylococcal infections. Clin Microbiol Infect 2008; 14: 3-9.

24. Spagnolo AM, Orlando P, Panatto D, Amicizia D, Perdelli F, Cristina ML. Staphylococcus aureus with reduced susceptibility to vancomycin in healthcare settings. J Prev Med Hyg 2014; 55: 137-44.

25. French GL. Bactericidal agents in the treatment of MRSA infections--the potential role of daptomycin. J Antimicrob Chemother 2006; 58: 1107-17.

26. Micek ST. Alternatives to vancomycin for the treatment of methicillin-resistant Staphylococcus aureus infections. Clin Infect Dis 2007; 45: 184-90.

\section{Publish in The International}

Arabic Journal of Antimicrobial Agents

The Journal is an open access peer-reviewed journal that publishes scientific papers about all aspects of antimicrobials. The journal will publish original research articles, reviews, brief reports and case reports dealing with basic and clinical antibacterial agents, antiviral, antiprotozoals, antituberculuous, antifungal and antihelminthes agents. All manuscripts must be prepared in English, and are subject to a rigorous and fair peer-review process. Accepted papers will immediately appear online. The journal aims to advance the knowledge, attitude and the research of chemotherapy in the Arabic world in cooperation with international, national scientific and public societies as well as research centers with similar aims and objectives. 